\title{
Flexión compuesta esviada en secciones de hormigón armado. Aproximación numérica
}

\section{Biaxial bending and axial load in reinforced concrete sections. Numerical aproach}

\author{
$\underline{\text { R. Lanti }}^{(*)}$, M. Martínez ${ }^{(* *)}$
}

RESUMEN

Las exigencias normativas relativas a la comprobación de los estados límites de servicio en estructuras de hormigón armado requieren del conocimiento de la tensión de tracción de la armadura en las secciones características. En situaciones de compresión o flexión compuesta esviada, la obtención de las coordenadas del plano de equilibrio con las que determinar deformación y tensión de cada punto de la sección transversal bajo solicitaciones no necesariamente de agotamiento, requiere de algún procedimiento numérico. Este trabajo presenta un método de búsqueda de la solución basado en la determinación de familias de diagramas momento-curvatura para la sección analizada y el axil de diseño exterior, y presenta la evaluación del mismo de manera experimental. El modelo es universal en cuanto a la geometría de la sección transversal, las solicitaciones exteriores, y los modelos constitutivos empleados para hormigón y acero.

Palabras clave: Flexión esviada, soportes de hormigón, tensión en servicio, métodos numéricos.

\section{ABSTRACT}

Perform serviceability requirements in reinforced concrete sections according to codes and standards need the knowledge of strain and stress in steel bars within the section. For general axial and biaxial bending, obtaining the response equilibrium plane of the transversal section is a problem that has to be solved by means of a numerical method. This paper exposes a general approach for this purpose, based on moment curvature families of diagrams for the external axial load, and presents an experimental evaluation. The method is universal in terms of to external loads, shape of the transversal sections, and also constitutive models of materials involved.

Keywords: Skew bending, reinforced concrete columns, service stress conditions, numerical methods.

(*) Doctor Arquitecto. Universidad de Alcalá, Alcalá de Henares, Madrid (España).

(**) Doctora Arquitecta. Universidad de Alcalá, Alcalá de Henares, Madrid (España).

Persona de contacto/Corresponding author: raul_lanti@hotmail.com (R. Lanti).

ORCID: https://orcid.org/oooo-0oo2-6406-3954 (R. Lanti); https://orcid.org/oooo-ooo3-4544-9517 (M. Martínez).

Cómo citar este artículo/Citation: Lanti, R.; Martínez, M. (2020). Flexión compuesta esviada en secciones de hormigón armado. Aproximación numérica. Informes de la Construcción, 72(558): e338. https://doi.org/10.3989/ic.69148.

Copyright: (c) 2020 CSIC. Este es un artículo de acceso abierto distribuido bajo los términos de la licencia de uso y distribución Creative Commons Reconocimiento 4.0 Internacional (CC BY 4.0). 


\section{INTRODUCCIÓN}

La literatura clásica de análisis estructural no estudia en general el problema de la determinación de las coordenadas del plano de equilibrio en secciones de hormigón armado sometidas a estados de compresión o flexión compuesta esviada. De manera tradicional, este problema se ha abordado mediante métodos basados en la determinación de la capacidad frente al agotamiento, procedimiento por el cual es establecido un límite superior y se garantiza la seguridad para las solicitaciones de diseño. En este punto el problema queda inacabado pues se carece de información a nivel de deformación y tensión de cada fibra de la sección transversal. En esta línea, autores como Pannel (1), Bresler (2), Grasser (3), Weber (4), Parme (5), o Morán (6) entre otros, han tratado el problema de la compresión o flexión compuesta esviada mediante la utilización de ábacos, formulas directas de dimensionamiento o comprobación, y diagramas de interacción en agotamiento. El método B de Bresler [1], sigue siendo de aplicación en los comentarios del código ACI-318 (7) y ampliamente utilizado en la práctica profesional habitual.

$$
\left(\frac{M_{x}}{M_{x o}}\right)^{\alpha}+\left(\frac{M_{y}}{M_{y o}}\right)^{\beta}=1.0
$$

En la actualidad, los modelos normativos vigentes establecen las bases de diseño con las que abordar el análisis a nivel de sección. Así, documentos como Eurocódigo 2 (8), la Instrucción EHE-o8 (9), y otros textos de referencia como el Model Code 90 (10), establecen los planos de agotamiento posibles de una sección solicitada frente a tensiones normales. Todos estos planos presentan una particularidad, y es que, al tratarse de planos de rotura, para todos ellos es conocido el punto en el que se alcanza el agotamiento de alguno de los materiales de la sección. Es decir cualquier plano de respuesta postulado que contenga un punto de agotamiento, (pivote en terminología normativa), es un plano de agotamiento de la sección. De este plano, y de manera directa es posible obtener sus coordenadas y determinar el mapa completo de deformaciones y tensiones de la sección analizada. El Prontuario Informático del Hormigón Estructural (11) resuelve en términos de aplicación informática este tipo de problema.

En situaciones en las que las solicitaciones exteriores no alcancen el valor de agotamiento de la sección, el plano solución de respuesta de la sección es desconocido, y no se dispone de ningún punto desde el planteamiento del problema con el que poder comenzar la búsqueda del plano solución. Todos los parámetros del plano solución son desconocidos y han de ser determinados.

Los modelos más recientes plantean la búsqueda de la solución exacta al problema mediante complejos modelos numéricos de difícil aplicación en la práctica habitual. Así, Bonet y Fernández (12), plantean un método numérico de integración de las tensiones en la zona no fisurada del hormigón en compresión mediante la división de esta zona en un número reducido de franjas mediante la cuadratura de Gauss-Legendre frente a la división clásica en un elevado número de celdillas o franjas. Otros como Barros (13) plantean una solución analítica para el diseño óptimo de secciones en estado límite último minimizando el coste de acero mediante el empleo de las que funciones tipo Heavyside para la caracterización de la deformación del hormigón. Gil-Martín (14) propone un mé- todo para el diseño óptimo desde un punto de vista de la cuantía (problema de mínimos) de secciones de hormigón armado. Desde el punto de vista del tratamiento de la sección en términos de momento-curvatura, Papanikolau (15) presenta un modelo basado en la integración de la tensión en el hormigón mediante el teorema de Green.

Por otra parte, y tratándose de hormigón estructural, un material cuyas características mecánicas se describen en términos estadísticos, resulta necesario plantearse el nivel de exigencia del modelo de análisis empleado en términos de su precisión frente a su dificultad numérica y operativa. Es necesario disponer de un algoritmo robusto que arroje soluciones suficientemente precisas con las que poder conocer la respuesta completa de una sección cualquiera en términos de deformación y tensión pero de reducida complejidad numérica. Conocidas las coordenadas del plano de equilirio, la aplicación de los modelos normativos en cuanto a las exigencias del cumplimientode los estados límites de servicio (control de la fisuración, flechas...) es trivial y segura.

Otros tipos estructurales pueden ser resueltos con un modelo similar al empleado en la resolución de secciones de hormigón estructural. Así, las placas base de soportes de perfiles de acero en su conexión a elementos de cimentación pueden abordarse con el método propuesto en este trabajo. En estos elementos, los pernos de anclaje resisten habitualmente solicitaciones de tracción (como respuesta a las demandas de axil y momentos según los dos ejes de referencia de la sección), así como esfuerzos cortantes. La comprobación de la integridad de los pernos ha de llevase a cabo necesariamente, tal como establecen los códigos normativos, mediante la interacción de ambas solicitaciones, para lo cual resulta necesario determinar el axil y el cortante que actúa en cada uno de los pernos de la placa base para las cargas de diseño.

\section{UN ALGORITMO PARA LA DETERMINACIÓN DEL PLANO DE EQUILIBRIO. MÉTODO DE LA DIFERENCIA MÍNIMA DE CURVATURAS (MDMC)}

Es posible determinar el plano de equilibrio de secciones simétricas solicitadas frente a un estado de compresión o flexión compuesta recta mediante la determinación del diagrama momento-curvatura de la sección para el axil de diseño. Conocida la curvatura como función del momento exterior, y la dirección de la fibra neutra que discurre según la normal a la dirección de la aplicación del vector momento exterior en secciones simétricas, es posible determinar la deformación y la tensión en cada una de las fibras de la sección trasversal.

En el supuesto de estados de solicitación más complejos $\left(\mathrm{N}, \mathrm{M}_{\mathrm{x}}, \mathrm{M}_{\mathrm{y}}\right)$ el procedimiento indicado no es de aplicación. No es posible abordar el problema general mediante la imposición a priori del rumbo de la fibra neutra, pues este valor es desconocido. No es posible por tanto abordar el problema mediante un tratamiento similar al utilizado en situaciones de flexión uniaxial y un único diagrama momento-curvatura para el axil de diseño. De llevar a cabo este análisis se garantizaría que, para cada par de momentos exteriores $\mathrm{M}_{\mathrm{x}}, \mathrm{M}_{\mathrm{y}}$, el rumbo de la fibra neutra de su plano solución discurriría según la dirección postulada, y que el axil interno movilizado a nivel de sección sería igual al exterior. Sin embargo, y a pesar 
de haber logrado mediante este planteamiento que la resultante de las componentes de los momentos internos movilizados sea de igual valor a la del momento exterior, cada una de las componentes de este momento interno no tendrían por que coincidir con los valores de las componentes del momento exterior. Luego, el plano determinado no sería un plano solución, y las tensiones obtenidas en las fibras características no serían las tensiones reales de respuesta de la sección analizada. Este hecho puede visualizarse con la interpretación del diagrama de interacción mostrado en la figura 1.

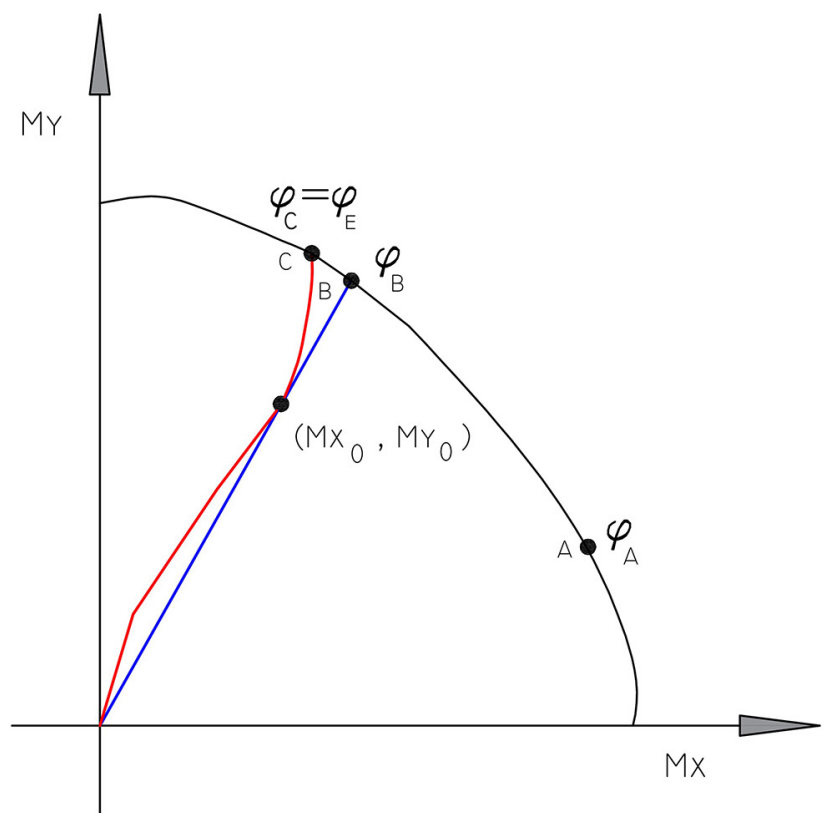

Figura 1. Diagrama general interacción $\left(\mathrm{M}_{\mathrm{x}}, \mathrm{M}_{\mathrm{y}}\right)$ para un axil concomitante de valor constante.

Sobre el diagrama de interacción mostrado en la figura 1 se ha representado un punto $\left(\mathrm{M}_{\mathrm{xo}}, \mathrm{M}_{\mathrm{yo}}\right)$ correspondiente a un estado de solicitación exterior. Sobre la figura, la línea azul representa el conjunto de pares de componentes $\left(\mathrm{M}_{\mathrm{x}}, \mathrm{M}_{\mathrm{y}}\right)$ cuya relación permanece constante (dirección del momento exterior constante) desde un estado de solicitación nulo hasta las solicitaciones de agotamiento, y es igual a la relación de las componentes del momento exterior. La línea roja representa el conjunto de valores $\left(\mathrm{M}_{\mathrm{x}}, \mathrm{M}_{\mathrm{y}}\right)$ para los que el giro de la fibra neutra permanece constante y coincide con el giro de equilibrio para las solicitaciones exteriores.
El modelo presentado en este trabajo plantea la determinación de una familia de diagramas momento-curvatura para todos los giros compatibles con el giro buscado y las solicitaciones exteriores entre los cuales poder elegir aquel diagrama momento-curvatura en el que, entrando con las dos componentes del momento de diseño se lea una única curvatura, o en su defecto, aquel diagrama momento-curvatura en el que la diferencia de curvaturas $\chi_{\mathrm{x}}, \chi_{\mathrm{y}}$ adopte un valor mínimo admisible.

Sobre la figura 1 se han incluido tres puntos de agotamiento: A, B, C. Para cada uno de estos puntos son conocidos los rumbos que adopta la fibra neutra, y para cada uno de estos giros es posible determinar un diagrama momento-curvatura (figura 2). En los diagramas mostrados en la figura 2 se incluyen las componentes del momento exterior según cada uno de los ejes de referencia de la sección.

Para el primero de los giros $\left(\varphi_{A}\right)$ puede observarse (figura 2A) el hecho de la imposibilidad de hallar la curvatura para la componente $\mathrm{M}_{\mathrm{yo}}$ del momento de diseño. De este hecho se extrae la conclusión de que este giro queda descartado como giro posible solución desde el origen del planteamiento del problema.

El segundo de estos puntos posee un giro de la fibra neutra compatible con la búsqueda del giro de equilibrio si bien en el caso mostrado (figura 2B) se trata de un giro que no satisface las condiciones para convertirse en solución puesto que la diferencia de curvaturas $\left(\Delta \chi_{\mathrm{B}}\right)$ es elevada.

Haciendo uso del diagrama momento-curvatura correspondiente al giro $\varphi_{\mathrm{c}}$, (figura $2 \mathrm{C}$ ), y las componentes del momento de diseño $\left(M_{x o}, M_{y 0}\right)$, puede observarse que la lectura de la curvatura es única $(\Delta \chi=0)$. Se trata del giro para el cual la sección analizada alcanza el equilibrio estricto, giro que no tiene por que coincidir necesariamente con la dirección normal a la dirección del vector momento exterior.

El método expuesto se basa en determinar un conjunto de diagramas momento-curvatura suficientemente amplio para de manera iterativa, poder seleccionar aquel en el que la diferencia de curvaturas para las dos componentes del momento exterior sea mínima. En este trabajo, las simulaciones que se presentan asumen la determinación de un diagrama momento-curvatura por cada grado de giro de la fibra neutra, lo cual

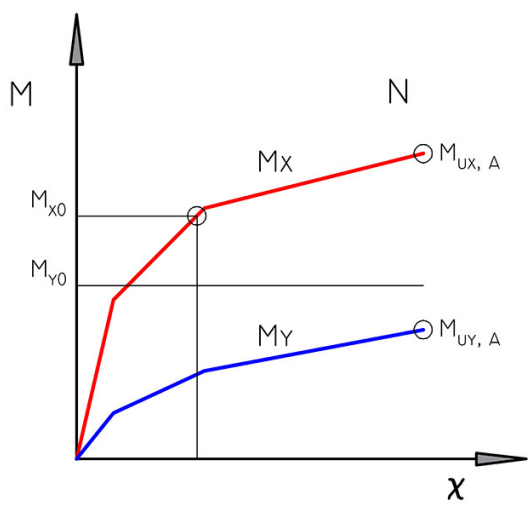

(A)

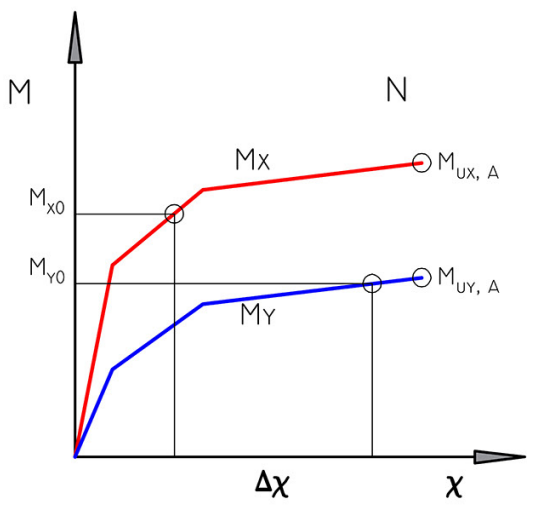

(B)

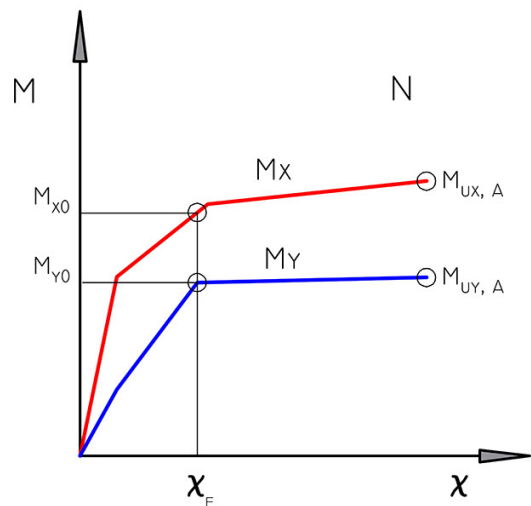

(c)

Figura 2. Lectura de la diferencia de curvaturas en tres diagramas momento curvatura para tres giros de la fibra neutra, sin lectura posible de diferencia de curvaturas (A), con lectura posible de diferencia de curvaturas (B), y con solución exacta de equilibrio (C). 
implica obtener una familia de 360 diagramas momento-curvatura para el axil de diseño. En este supuesto el error máximo que se produce en la estimación de la dirección de la traza del plano de equilibrio es 0,50 frente a la solución exacta.

\section{EVALUACIÓN EXPERIMENTAL DEL MDMC}

\subsection{Metodología de trabajo}

Se lleva a cabo un ensayo destructivo controlado en laboratorio de un soporte corto de hormigón armado sometido a un estado de compresión compuesta esviada. Tanto la preparación del prototipo como el desarrollo de todas las fases del ensayo experimental se llevan a cabo en las instalaciones del Instituto de Ciencias de la Construcción Eduardo Torroja. Se observan y analizan los planos de respuesta de una sección de referencia del prototipo y se comparan frente a los resultados que resultan de llevar a cabo la reproducción numérica de este ensayo experimental mediante un software propio desarrollado en el lenguaje de programación orientado a objetos c\# de la plataforma .Net, en el que se ha implementado el MDMC.

\subsection{Descripción del prototipo ensayado experimentalmente}

La descripción geométrica y definición de armadura del prototipo ensayado se muestran en la figura 3.
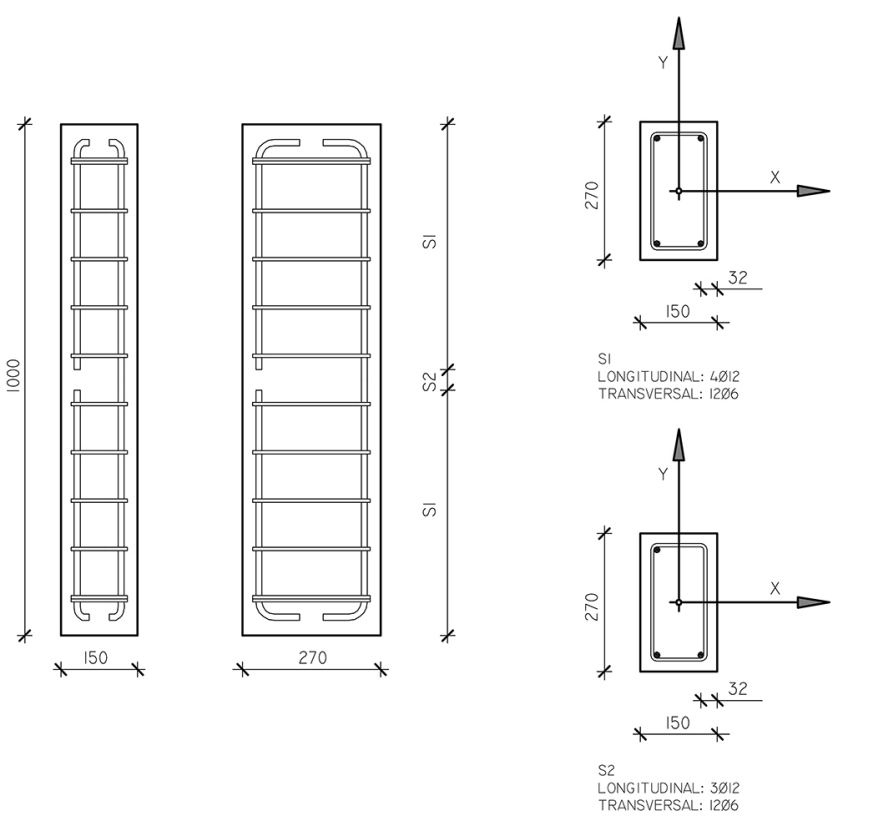

Figura 3. Diseño del prototipo de hormigón ensayado.

Puede observarse que la probeta consta de dos secciones a lo largo de su directriz. La sección tipo ( $\mathrm{S} 1)$ presenta cuatro barras de armadura longitudinal ancladas en los extremos de la probeta mediante doblado en patilla. La sección central (S2) se ha considerado la sección de referencia del trabajo y es la sección instrumentada. En esta sección se interrumpe una de las cuatro barras longitudinales de armadura con el fin de dotarla de una asimetría mecánica notable. La intención de esta singularidad en la armadura es doble: en primer lugar se plantea un problema de difícil solución mediante los modelos clásicos, aptos para secciones con distribuciones regulares de armadura y secciones transversales con geometrías regula- res, lo cual justifica la necesidad de la implementación del MDMC. En segundo lugar se pretende condicionar la respuesta del plano de equilibrio en cada escalón de carga y observar la evolución del giro que adopta la fibra neutra en equilibrio en relación a la dirección de la excentricidad exterior, y poder así constatar la ausencia de perpendicularidad entre ambas direcciones.

Se ha empleado un hormigón HA-30, y dada la alta sensibilidad de la cuestión investigada se ha llevado a cabo un ensayo destructivo de una probeta cilíndrica de 15x30 cm, obteniéndose la curva tensión-deformación representada en la figura 4 con la línea azul.

Es preciso llevar a cabo un ajuste numérico de la curva tensión-deformación obtenida de manera experimental mediante la expresión incluida en los comentarios de la Instrucción EHE-08 [2], modificando la rama descendente y rectificando la caída abrupta de tensión por el fallo de la probeta durante el propio ensayo.

$$
\sigma_{c}=\frac{k \eta-\eta^{2}}{1+(k-2) \eta} f_{c m} \quad \text { para } \varepsilon_{c} \leq \varepsilon_{c u}
$$

En el caso expuesto en este trabajo, la curva que resulta de este ajuste numérico se representa con la línea roja de la figura 4, para la cual se ha obtenido un valor de $\varepsilon_{c u}=2,536 \%$ y un valor de tensión máxima $\sigma=31,61 \%$.

Se ha empleado un acero B500SD, cuya caracterización mecánica se hace según lo establecido en la Instrucción EHE-o8.

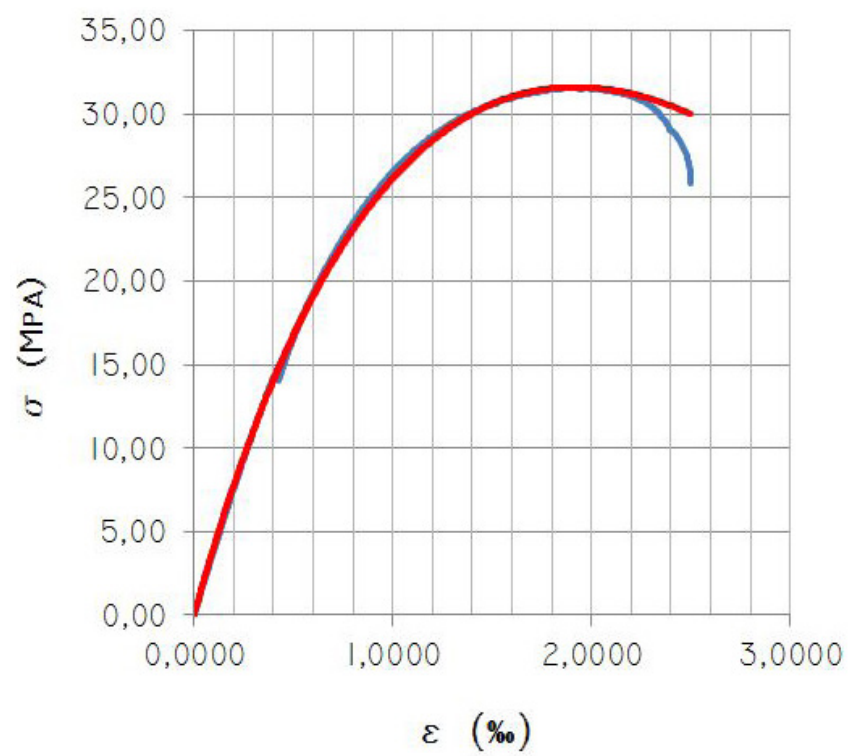

Figura 4. Curva tensión-deformación del hormigón del prototipo ensayado. La curva azul representa la lectura de las deformaciones frente a las tensiones durante el ensayo destructivo de la probeta. La curva roja muestra la curva tensióndeformación ajustada mediante las expresiones incluidas a tal efecto en la Instrucción EHE-o8.

\subsection{Descripción del ensayo experimental}

El estado exterior de compresión compuesta esviada al que se somete al soporte se logra mediante la aplicación de un axil exterior y una excentricidad constante en el eje $\mathrm{Y}$ de la sec- 
ción transversal $\left(\mathrm{M}_{\mathrm{x}}\right)$, $\mathrm{y}$ un momento $\mathrm{M}_{\mathrm{y}}$ de valor creciente desde un valor nulo hasta el lograr su agotamiento. El procedimiento para lograr estos estados de solicitación exterior se lleva a cabo en dos fases.

En la primera fase se aplica un axil exterior de valor $40,51 \mathrm{kN}$ y una excentricidad $e_{\mathrm{y}}=195 \mathrm{~mm}$ sobre el eje de mayor inercia de la sección transversal, lo cual induce un momento flector en el centro de gravedad de la sección de referencia del soporte (S2) de valor $M_{\mathrm{x}}=7,90 \mathrm{kN} \cdot \mathrm{m}$. El axil excéntrico de compresión se logra mediante la tracción aplicada a una barra de acero de límite elástico $f_{\mathrm{y}}=500 \mathrm{MPa}$ y $16 \mathrm{~mm}$ de diámetro situada a una distancia constante del centro de gravedad de la sección transversal del soporte (figura 5).

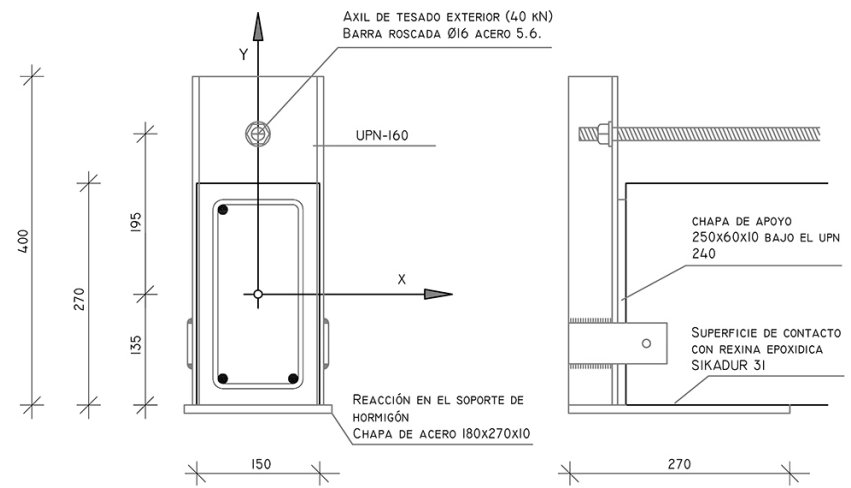

Figura 5. Esquema de aplicación del axil sobre el eje Y de la sección transversal en el ensayo.

En la segunda fase del ensayo se aplica una carga perpendicular a la directriz del soporte de hormigón, según el eje $\mathrm{X}$ de la sección transversal a una velocidad constante de $5 \mathrm{kN} / \mathrm{min}$. hasta la rotura del prototipo. La figura 6 muestra un instante de la materialización de esta fase del ensayo.

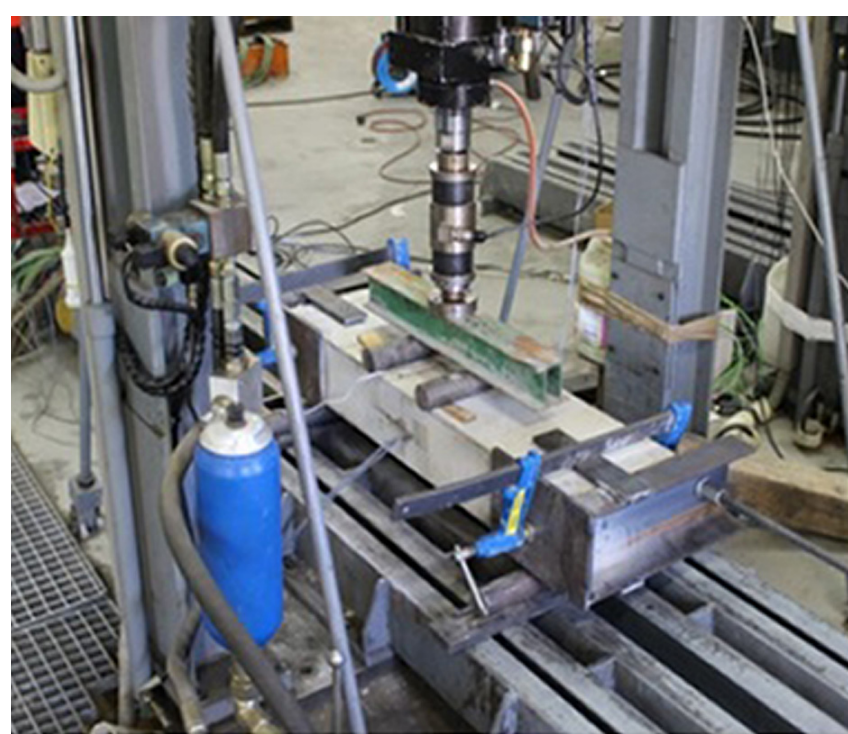

Figura 6. Vista del soporte de hormigón situado para la aplicación de la carga exterior en el plano débil de la sección transversal y el axil de tesado exterior aplicado.

\subsection{Instrumentación del prototipo}

Se instrumentan las fibras características (barras de armadura y puntos medios de las caras del prototipo) en la sección de referencia. En cada barra de armadura se instalan dos instru- mentos para obtener medidas redundantes de las deformaciones. Cada uno de los diez instrumentos instalados se conecta con un sistema de lectura e interpretación con el que poder registrar la deformación que experimentan en cada instante de tiempo. Concluido el ensayo se dispone de un registro continuo de deformación longitudinal frente a las solicitaciones exteriores aplicadas.

\subsection{Resultados observados}

Para la interpretación de los planos de respuesta de la sección se han escogido un total de 20 estados concretos de solicitación exterior para los que se han determinado los planos de equilibrio. Se ha partido de una carga exterior aplicada de valor $29,25 \mathrm{kN}$, y se ha incrementado en cada escalón de carga en un valor de $2,89 \mathrm{kN}$ hasta alcanzar el valor de $90,00 \mathrm{kN}$.

El valor inicial de carga vertical considerada se debe a que para este valor el momento inicial $\mathrm{M}_{\mathrm{yo}}$ representa el $3 \%$ (una fracción reducida) del momento total en esa misma sección para la carga elegida, minimizándose las diferencias existentes entre los planos de respuesta observados y los reales (que incluyen la deformación correspondiente al peso propio del prototipo). Es preciso tomar en consideración que el equipo de lectura arroja información solamente para los incrementos de carga impuestos, no quedando registradas las deformaciones iniciales existentes en todas las fibras de la sección de referencia debido al peso propio de rodillos y perfiles de aplicación de la carga y del peso propio del soporte ensayado. La interpretación de los resultados se hace en coherencia con la figura 7.

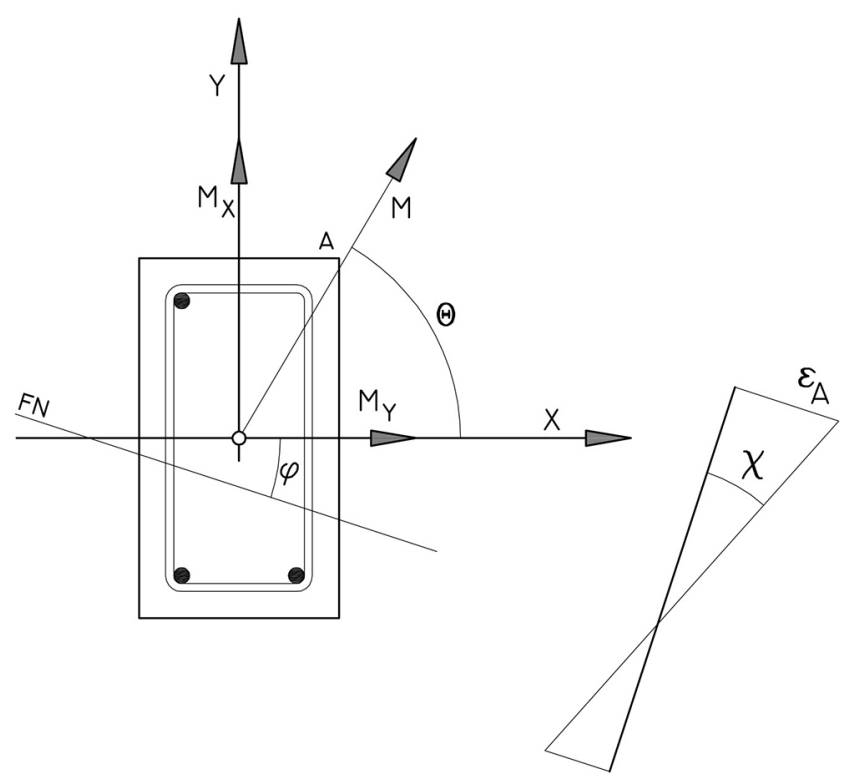

Figura 7. Convenio de notación de dirección de aplicación del vector momento en el ensayo, y descripción de los parámetros del plano de equilibrio.

La tabla 1 recoge las solicitaciones exteriores consideradas. Se observa que los valores de axil y momento flector sobre el eje de mayor inercia de la sección $\left(\mathrm{N}, \mathrm{M}_{\mathrm{x}}\right)$ permanecen constantes al tiempo que el valor de $\mathrm{M}_{\mathrm{y}}$ aumenta, y se muestra el valor de la carga exterior aplicada por el equipo de carga $\left(Q_{x}\right)$ que induce dicho momento $\mathrm{M}_{\mathrm{y}}$. Se han determinado mediante la interpretación geométrica de las deformaciones de las fibras instru- 
Tabla 1. Valores de solicitaciones exteriores considerados sobre la sección de referencia para el análisis de los resultados.

\begin{tabular}{|c|c|c|c|c|c|c|c|c|c|c|}
\hline $\begin{array}{l}\text { Escalón } \\
\text { de Carga }\end{array}$ & $\begin{array}{l}\text { Axil } \\
(k N)\end{array}$ & $\underset{(\mathbf{k N} \cdot \mathbf{m})}{\mathbf{M}_{\mathrm{x}}}$ & $\underset{(\mathbf{k N} \cdot \mathbf{m})}{\mathbf{M}_{\mathbf{y}}}$ & $\begin{array}{c}\mathbf{Q}_{\mathrm{x}} \\
(\mathbf{k N})\end{array}$ & $\begin{array}{c}\theta \\
\left({ }^{\circ}\right)\end{array}$ & $\begin{array}{c}\boldsymbol{\varphi} \\
\left(^{\circ}\right)\end{array}$ & $\underset{\substack{x \\
(1 / \mathbf{k m})}}{ }$ & $\begin{array}{l}\text { Prof. } \\
\text { (mm) }\end{array}$ & $\begin{array}{c}\varepsilon_{\mathrm{A}} \\
(\%)\end{array}$ & $\begin{array}{c}\sigma_{\mathrm{A}} \\
(\mathbf{M P a})\end{array}$ \\
\hline 1 & 40,51 & 7,90 & 4,52 & 29,25 & 60,21 & 75,98 & 12,590 & 124,12 & $-1,102$ & 27,37 \\
\hline 2 & 40,51 & 7,90 & 4,96 & 32,14 & 57,89 & 76,99 & 13,507 & 135,68 & $-1,137$ & 27,74 \\
\hline 3 & 40,51 & 7,90 & 5,39 & 35,04 & 55,69 & 78,06 & 14,562 & 150,31 & $-1,173$ & 28,12 \\
\hline 4 & 40,51 & 7,90 & 5,82 & 37,93 & 53,60 & 78,93 & 15,551 & 164,57 & $-1,206$ & 28,46 \\
\hline 5 & 40,51 & 7,90 & 6,26 & 40,82 & 51,61 & 79,78 & 16,667 & 180,61 & $-1,243$ & 28,79 \\
\hline 6 & 40,51 & 7,90 & 6,69 & 43,71 & 49,73 & 80,44 & 17,643 & 194,85 & $-1,276$ & 29,08 \\
\hline 7 & 40,51 & 7,90 & 7,13 & 46,61 & 47,95 & 80,97 & 18,525 & 207,93 & $-1,306$ & 29,33 \\
\hline 8 & 40,51 & 7,90 & 7,56 & 49,50 & 46,26 & 81,46 & 19,450 & 221,56 & $-1,337$ & 29,55 \\
\hline 9 & 40,51 & 7,90 & 7,99 & 52,39 & 44,66 & 82,09 & 20,750 & 241,69 & $-1,380$ & 29,87 \\
\hline 10 & 40,51 & 7,90 & 8,43 & 55,29 & 43,15 & 82,50 & 21,692 & 256,24 & $-1,411$ & 30,08 \\
\hline 11 & 40,51 & 7,90 & 8,86 & 58,18 & 41,71 & 82,99 & 22,983 & 275,79 & $-1,456$ & 30,34 \\
\hline 12 & 40,51 & 7,90 & 9,30 & 61,07 & 40,36 & 83,39 & 23,967 & 293,51 & $-1,496$ & 30,57 \\
\hline 13 & 40,51 & 7,90 & 9,73 & 63,96 & 39,07 & 83,74 & 25,165 & 310,28 & $-1,532$ & 30,74 \\
\hline 14 & 40,51 & 7,90 & 10,16 & 66,86 & 37,86 & 84,18 & 26,551 & 333,42 & $-1,581$ & 30,95 \\
\hline 15 & 40,51 & 7,90 & 10,60 & 69,75 & 36,70 & 84,54 & 27,910 & 355,58 & $-1,631$ & 31,13 \\
\hline 16 & 40,51 & 7,90 & 11,03 & 72,64 & 35,61 & 85,15 & 30,227 & 399,15 & $-1,712$ & 31,36 \\
\hline 17 & 40,51 & 7,90 & 11,47 & 75,54 & 34,57 & 85,59 & 31,992 & 440,97 & $-1,763$ & 31,46 \\
\hline 18 & 40,51 & 7,90 & 11,90 & 78,43 & 33,58 & 86,30 & 34,808 & 524,26 & $-1,843$ & 31,56 \\
\hline 19 & 40,51 & 7,90 & 12,33 & 81,32 & 32,64 & 87,04 & 37,440 & 658,90 & $-1,888$ & 31,60 \\
\hline 20 & 40,51 & 7,90 & 13,64 & 90,00 & 30,09 & 88,01 & 41,130 & 976,26 & $-1,953$ & 31,59 \\
\hline
\end{tabular}

mentadas los planos de respuesta para cada uno de los 20 estados de solicitación exterior. Se incluye igualmente en la tabla 1 el valor de la deformación y la tensión de compresión en el vértice A, (punto de mayor deformación de compresión en la sección), determinada mediante la utilización del modelo constitutivo descrito para el hormigón en la figura 4.

\subsection{Análisis numérico}

Para los 20 estados de solicitación exterior considerados en el análisis experimental se han determinado de manera numérica mediante un software propio desarrollado y el MDMC las coordenadas de los planos de respuesta. Los valores que des-

Tabla 2. Coordenadas del plano de equilibrio obtenidas en el análisis numérico para los veinte niveles de carga establecidos.

\begin{tabular}{|c|c|c|c|c|c|}
\hline $\begin{array}{c}\text { Escalón } \\
\text { de Carga }\end{array}$ & $\begin{array}{c}\boldsymbol{\varphi} \\
\mathbf{(}^{\circ}\end{array}$ & $\begin{array}{c}\boldsymbol{X} \\
(\mathbf{1} / \mathbf{k m})\end{array}$ & $\begin{array}{c}\text { Prof. } \\
(\mathbf{m m})\end{array}$ & $\begin{array}{c}\mathbf{\varepsilon}_{\mathbf{A}} \\
\mathbf{( \% )}\end{array}$ & $\begin{array}{c}\mathbf{\sigma}_{\mathbf{A}} \\
(\mathbf{M P a})\end{array}$ \\
\hline 1 & 66 & 7,17 & 38.00 & 0,613 & 19.36 \\
\hline 2 & 69 & 8,03 & 37,95 & 0,646 & 20.06 \\
\hline 3 & 71 & 9,01 & 38,01 & 0,693 & 21.05 \\
\hline 4 & 73 & 9,98 & 37,81 & 0,732 & 21.80 \\
\hline 5 & 74 & 10,91 & 38,9 & 0,768 & 22.46 \\
\hline 6 & 75 & 11,68 & 38,91 & 0,800 & 23.06 \\
\hline 7 & 76 & 12,61 & 38,37 & 0,845 & 23.80 \\
\hline 8 & 77 & 13,84 & 39,01 & 0,892 & $24 \cdot 57$ \\
\hline 9 & 78 & 14,89 & 38,87 & 0,931 & 25.14 \\
\hline 10 & 79 & 16,21 & 39,17 & 0,976 & 25.78 \\
\hline 11 & 79 & 16,75 & 39,15 & 1,009 & 26.23 \\
\hline 12 & 80 & 18,35 & 39,17 & 1,067 & 26.94 \\
\hline 13 & 80 & 18,93 & 39,24 & 1,099 & 27.35 \\
\hline 14 & 80 & 19,52 & 39,33 & 1,132 & 27.69 \\
\hline 15 & 81 & 21,48 & 39,26 & 1,202 & 28.42 \\
\hline 16 & 81 & 22,02 & 39,26 & 1,231 & 28.68 \\
\hline 17 & 81 & 22,58 & 39,33 & 1,261 & 28.95 \\
\hline 18 & 82 & 25,65 & 39,19 & 1,382 & 29.88 \\
\hline 19 & 82 & 26,38 & 39,49 & 1,413 & 30.09 \\
\hline 20 & 82 & 29,37 & 40,23 & 1,551 & 30.82 \\
\hline
\end{tabular}

criben los planos obtenidos, así como la deformación y la tensión en el vértice A de la sección, en coherencia con el criterio establecido en la figura 7 muestran en la tabla 2.

La figura 8 muestra a modo de ejemplo la salida del software para la simulación numérica del escalón 10 de solicitación exterior. En esta figura es posible visualizar el giro que adopta la fibra neutra en equilibrio así como los perfiles de tensiones normales de respuesta en hormigón y acero. La salida del software se completa con el diagrama de interacción para el axil de diseño $(40,51 \mathrm{kN})$ y los diagramas momento-curvatura para el giro de equilibrio, en los que se puede observar como las curvaturas referidas a los ejes $\mathrm{x}$, y para las compo-

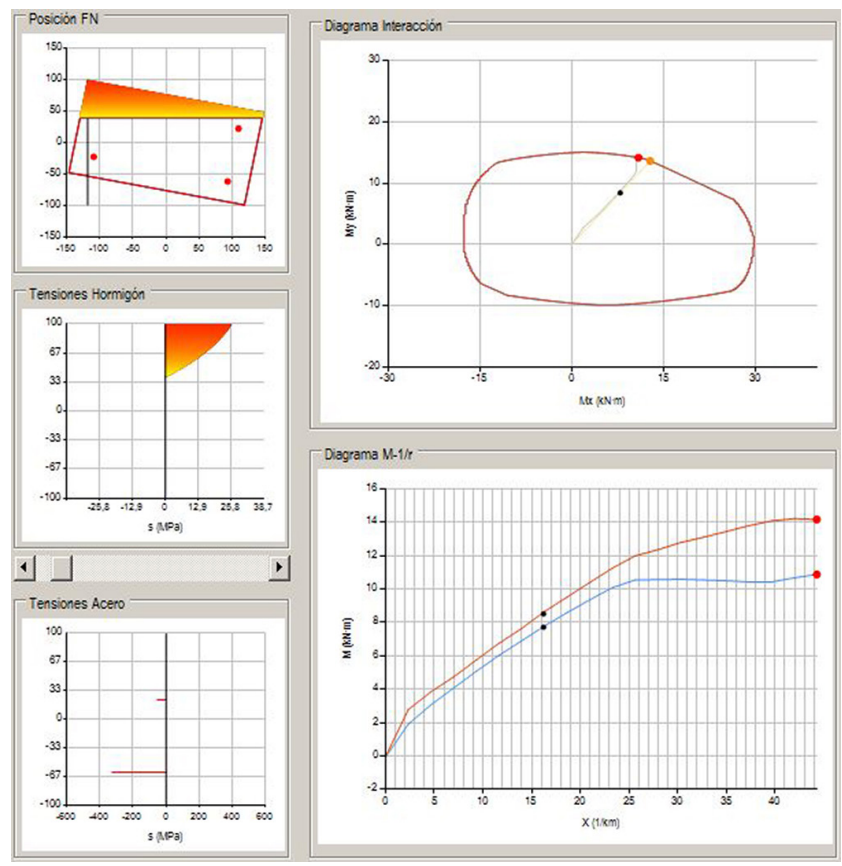

Figura 8. Análisis a nivel de sección correspondiente al escalón de solicitación exterior 10. 
nentes del momento exterior de diseño $\left(\mathrm{M}_{\mathrm{xo}}, \mathrm{M}_{\mathrm{yo}}\right)$, coinciden tal como establece el MDMC.

\subsection{Discusión de los resultados}

Se ha observado en laboratorio que la carga de rotura alcanzada supera sensiblemente a la carga en rotura determinada de manera numérica y teórica, hallándose un incremente de la primera respecto a la segunda del $17 \%$. Este incremento puede deberse a las siguientes causas:

- En el análisis numérico llevado a cabo no se ha tomado en consideración la capacidad del hormigón para resistir solicitaciones de tracción.

- Debido a la posición de la carga según el eje X, y puesto que esta se lleva al soporte de hormigón mediante un perfil de acero apoyado en dos rodillos, es posible que en fases próximas a la rotura, se produjera un cierto arco de descarga, y una fracción de la carga aplicada fuera conducida directamente mediante bielas a los apoyos, reduciéndose el momento exterior en la sección de referencia.

- El modelo de cálculo de equilibrio de la sección en cuanto a la resultante de esfuerzos internos no contempla otros posibles mecanismos resistentes adicionales que abundan en el aumento de capacidad real de la sección.

Se ha observado que los resultados obtenidos de manera experimental sobre el prototipo para los veinte estados de carga establecidos en cuanto a las coordenadas de los planos de equilibrio son similares a los planos determinados de manera numérica mediante la aplicación del modelo propuesto en este trabajo (MDMC). En la figura 9 se muestran las tra-

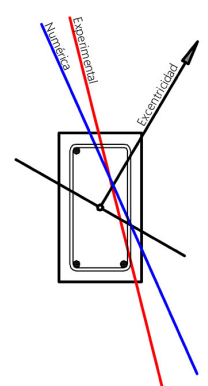

(1)
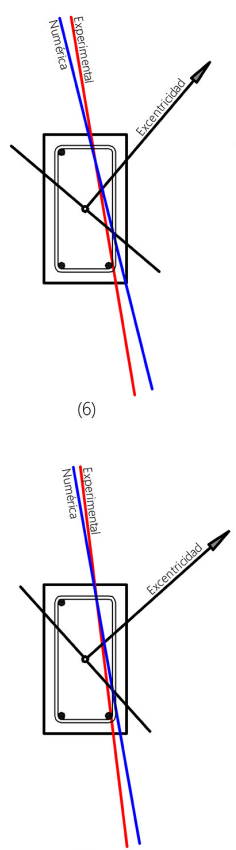

(11)

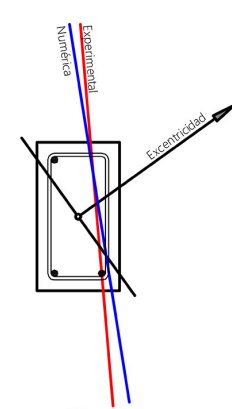

(16)

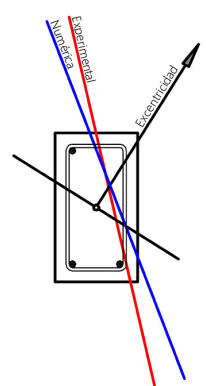

(2)

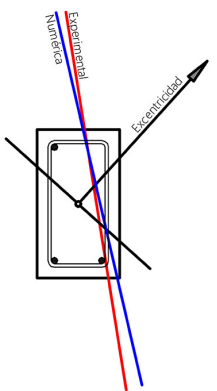

(7)

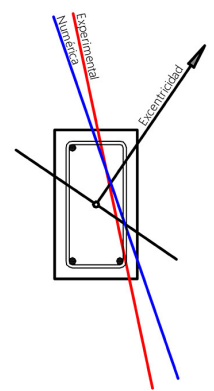

(3)

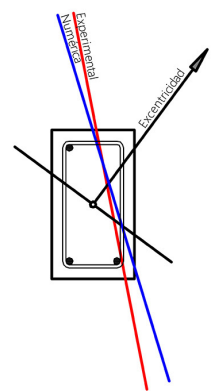

(4)

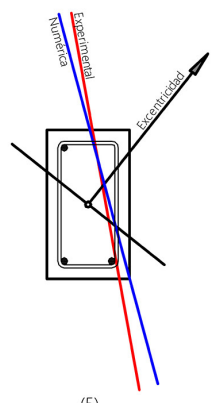

(5)
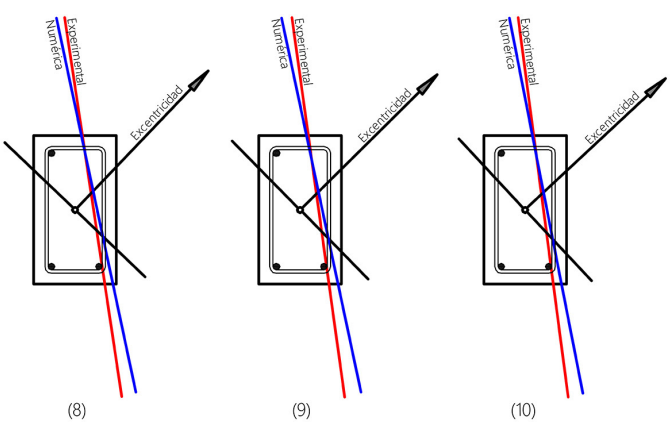

(10)

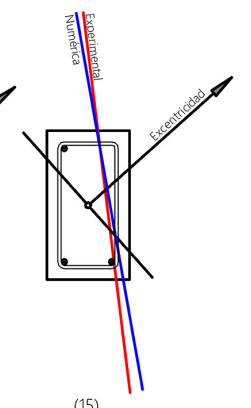

(15)

(14)
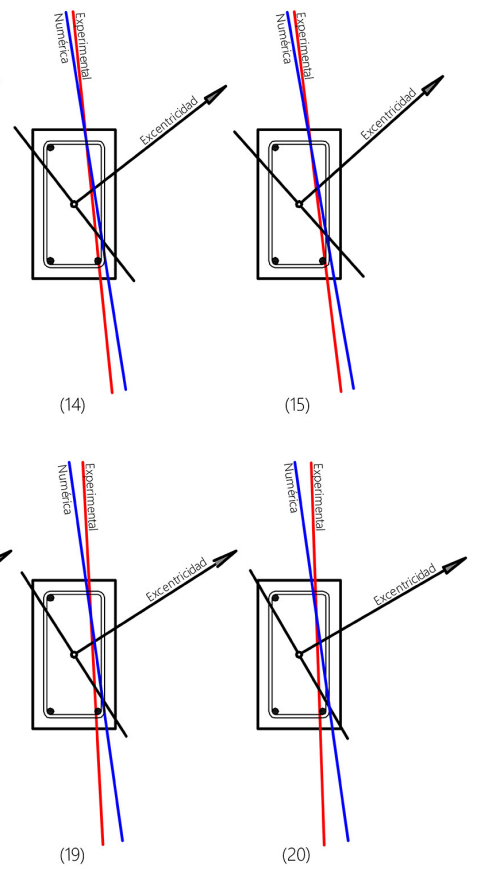

Figura 9. Posición de la fibra neutra obtenida de manera experimental y numérica para los veinte escalones de carga analizados. 
zas de los planos de respuesta (fibras neutras) para los veinte estados de carga. En rojo se representan las soluciones observadas de manera experimental. Las líneas azules corresponden a las soluciones determinadas de manera numérica (MDMC).

Con la intención de mostrar la variación que experimenta la relación entre el rumbo que adopta la fibra neutra en equilibrio y la dirección normal a la dirección de la excentricidad exterior, en la figura 9 se ha incluido la dirección del vector momento exterior aplicado en cada uno de los estados de carga analizados. La evolución de esta relación frente al ratio de utilización de la sección se muestra en la figura 10.

Puesto que no es posible determinar de manera numérica estados de equilibrio para solicitaciones mayores a las solicitaciones de agotamiento teórico, en la figura 10 se ha atribuido como ratio 1 (estado de agotamiento) al que se determina de manera teórica mediante el método numérico propuesto y las bases de cálculo establecidas en la Instrucción EHE-o8.

\section{CONCLUSIONES}

Del análisis de los resultados experimentales y numéricos presentados en este trabajo es posible establecer las siguientes conclusiones:

- Se ha constatado la dificultad que supone tratar de materializar ensayos de elementos estructurales de hormigón armado sometidos a estados de compresión o flexión compuesta esviada en situaciones de no agotamiento (ELS). Existe un número elevado de variables que abundan en este hecho y que pueden distorsionar la lectura de los instrumentos instalados en los prototipos y su correlación con los valores teóricos esperados. Factores como la tensión resistente del hormigón frente a solicitaciones de tracción, el posible no cumplimiento de la hipótesis de Bernouilli-Navier, (modelos energéticos frente a los modelos de equilibrio de solicitaciones y conservación del plano de la sección), mecanismos resistentes adicionales no contemplados en el modelo teórico, o la no consideración de las deformaciones iniciales en la sección transversal por efecto del peso propio del prototipo y los medios de aplicación de la carga, pueden provocar pequeñas diferencias entre los resultados observados de manera experimental y los determinados de manera numérica.

- Se observa que en todos los casos de carga analizados, el giro determinado de manera numérica para la fibra neutra en equilibrio ha sido inferior al giro observado de manera experimental, lo cual podría deberse a la no consideración de algún mecanismo resistente no contemplado en el modelo teórico. Esta hipótesis es coherente con la diferencia entre la carga real de agotamiento alcanzada en laboratorio frente a la teórica esperada, un $17 \%$.

- Si se comparan los planos de respuesta obtenidos experimentalmente frente a los determinados de manera numérica, es posible afirmar que el modelo propuesto (MDMC)

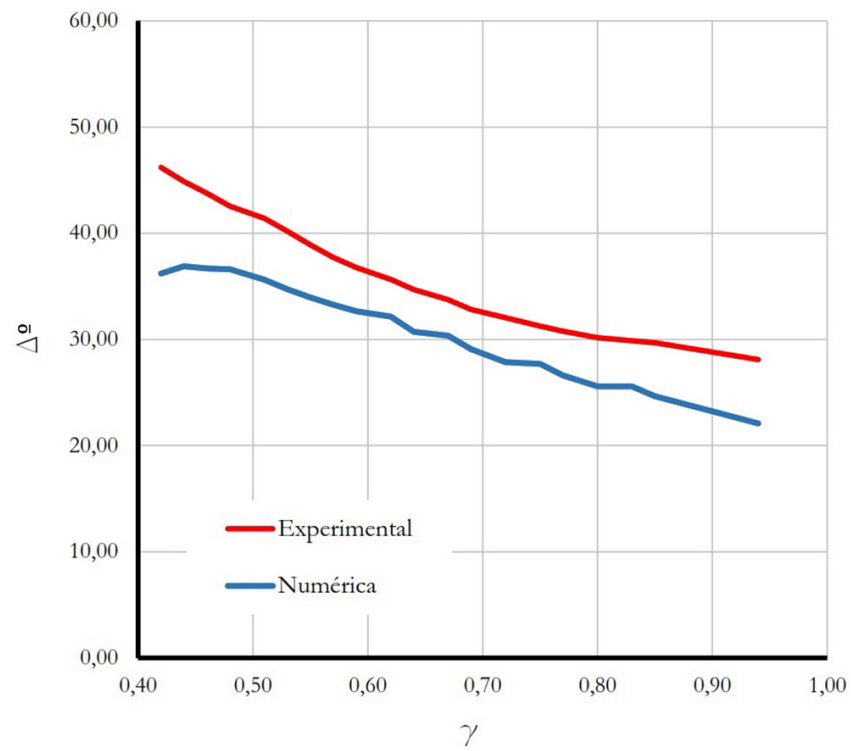

Figura 10. Diferencia en grados entre la dirección normal a la dirección de excentricidad exterior para las soluciones determinadas de manera experimental y numérica frente al ratio de utilización de la sección analizada.

es adecuado para la predicción de la respuesta de una sección de hormigón armado sometida a un estado de solicitación de compresión o flexión compuesta esviada. La figura 9 muestra para cada uno de los veinte estados de solicitación analizada la traza del plano de equilibrio (fibra neutra) así como la dirección de la aplicación del vector momento exterior. Se observa que a pesar de haberse encontrado diferencias entre ambas soluciones, se ha estimado de manera adecuada la tendencia del giro adoptado por la fibra neutra así como su profundidad y curvatura de respuesta en todos los rangos de utilización de la sección. La diferencia entre los dos giros (experimental y numérico) de la fibra neutra ha sido en todos los casos inferior a $10^{\circ}$

- Dada la configuración de la sección de referencia del prototipo analizado en la que se elimina una barra de armadura longitudinal, es posible afirmar que el modelo propuesto resulta eficaz en secciones genéricas carentes de simetría mecánica, con distribuciones no regulares de armadura.

- Se ha constatado la ausencia de perpendicularidad entre el giro que adopta la fibra neutra de una sección en equilibrio sometida a solicitaciones normales frente a la dirección de aplicación del vector momento exterior, y se ha observado que las mayores diferencias se encuentra en umbrales iniciales de carga (zonas de la sección en servicio), figura 10. Este hecho justifica la necesidad de emplear el modelo presentado en el análisis de secciones de hormigón estructural, pues cualquier intento de aproximación de la solución mediante los modelos clásicos conduciría a errores significativos en la estimación de la tensión en las fibras características, y por extensión en la aplicación de los códigos en cuanto al cumplimiento de los estados límite de servicio (fisuración y deformación). 


\section{REFERENCIAS}

(1) Pannell, F.N. (1959). Biaxially Loaded Reinforced Concrete Columns. ACI Journal, Proceedings, ASCE V.85, ST6: $47-54$.

(2) Bresler, B. (1960). Design Criteria for Reinforced Columns Under Axial Load and Biaxial Bending. ACI Journal, Proceedings, 57: 481-490.

(3) Rüsch, H., Grasser, E., Rao, P. (1962). Principes de calcul du béton armé sous des états de contraintes monoaxiaux. Bulletin $n^{0} 36$ of CEB.

(4) Weber, D.C. (1966). Ultimate Strength Design Charts for Columns with Biaxial Bending. ACI Journal, Proceedings, 63: 1205-1320, Disc., 1538-1586.

(5) Parme, A., Nieves, J., Gouwens, A. (1966). Capacity of Reinforced Rectangular Columns Subjected to Biaxial Bending. ACI Journal, Proceedings, 63: 911-923.

(6) Morán, F. (1972). Design of Reinforced Concrete Sections Under Normal Loads and Stresses in the Ultimate State. CEB Bulletin d'Information No83.

(7) ACI Committee 318, American Concrete Institute. (2014). Building Code Requirements for Structural Concrete ACI-318-14.

(8) Comité Europeo de Normalización (1992). Eurocódigo 2, Proyecto de Estructuras de Hormigón. AENOR.

(9) Ministerio de Fomento, Comisión Permanente del Hormigón (2008). Instrucción de Hormigón Estructural EHE-o8.

(10) CEB-FIP Model Code (1990). Comité Euro-International du Beton.

(11) Corres, H., Pérez, A., Martínez, J.L., López, J.C. (2008). Prontuario Informático del Hormigón Estructural EHE-o8. Madrid: Instituto Español del Cemento y sus Aplicaciones.

(12) Fernández, M.A., Bonet, J.L., Miguel Sosa, P.F. (2000). Integración de tensiones en secciones de hormigón sometidas a flexo compresión esviada. Revista Internacional de Métodos Numéricos para Cálculo y Diseño en Ingeniería, 16(2): 209-225.

(13) Barros, M., Barros, A. and Ferreira, C. (2004). Closed form solution of optimal design of rectangular reinforced concrete sections. Engineering Computations, 21(7): 761-776. https://doi.org/10.1108/02644400410565298

(14) Gil-Martín, L.M., Hernández-Montes, E., Aschheim, M. (2010). Optimal reinforcement of RC columns for biaxial bending. Material and Structures, 43(9): 1245-1256. https://doi.org/10.1617/s11527-009-9576-x

(15) Papanikolaou, V.K. (2012). Analysis of arbitrary composite sections in biaxial bending and axial load. Computers and Structures, 98-99: 33-54. https://doi.org/10.1016/j.compstruc.2012.02.004 